\title{
APPLICABILITY WITH PRESERVATION OF BOTH CURVATURES*
}

BY W. C. GRAUSTEIN

1. Introduction. In his celebrated memoir of 1867 , Bonnett proved that there is in general no surface applicable to a given surface with preservation of both the total and mean curvatures, but that in certain exceptional cases there exists a unique surface or a one-parameter family. The primary purpose of this note is to exhibit conditions of simple form characterizing the second of these exceptional cases. The method of treatment applies equally well to the general case.

2. Necessary and Sufficient Condition. It is well known that a necessary condition that a surface $S$ admit $\infty^{1}$ surfaces applicable to it with preservation of both curvatures is that the surface $S$ be isometric. $t$ Accordingly, we can assume that $S$ is an isometric surface, referred to its lines of curvature, and that the parameters are isometric. The linear element of $S$ is then of the form

$$
d s^{2}=\lambda\left(d u^{2}+d v^{2}\right)
$$

* Presented to the American Mathematical Society, September 7, 1923.

$\dagger$ Mémoire sur la théorie des surfaces applicables sur une surface donnée, Journal de L'École Polytechnique, vol. 42 (1867), pp. 72 et seq.

\$ This fact appears to have been discovered first by Raffy, Sur une classe nouvelle de surfaces isothermiques et sur les surfaces déformables sans alteration des courbures principales, BULLETIN DE LA Société DE France, vol. 21 (1893), pp. 70-72. His proof, however, is faulty, in that he bases part of it on the incorrect statement, made by Caronnet, that every $W$-surface applicable to a surface of revolution is isometric. If that statement were correct, every helecoidal surface would be isometric, which is not the case. A valid proof is given by Hazzidakis, Biegung mit Erhaltung der Hauptkrümmungsradien, JouRNAL FÜR MATHEMATIK vol. 117 (1897), p. 46. 
and the Codazzi equations become

$$
\frac{\partial e}{\partial v}=\frac{e+g}{2 \lambda} \frac{\partial \lambda}{\partial v}, \frac{\partial g}{\partial u}=\frac{e+g}{2 \lambda} \frac{\partial \lambda}{\partial u},
$$

where $e, f(=0), g$ are the differential coefficients of the second order. Recalling that $1 / r_{1}=e / \lambda, 1 / r_{2}=g / \lambda$, and setting

$$
\frac{1}{r_{1}}+\frac{1}{r_{2}}=2 M, \frac{1}{r_{1}}-\frac{1}{r_{2}}=2 N,
$$

we find that equations (2) can be rewritten in the forms* (3) $\frac{\partial M}{\partial u}=N \frac{\partial \log \lambda N}{\partial u}, \frac{\partial M}{\partial v}=-N \frac{\partial \log \lambda N}{\partial v}$.

If there exists a surface $S^{\prime}$ applicable to $S$ with preservation of both curvatures, then

$$
e^{\prime} g^{\prime}-f^{\prime 2}=e g, \quad e^{\prime}+g^{\prime}=e+g \text {. }
$$

Hence it can be shown that

(4) $e^{\prime}=e-\frac{2 \lambda N}{1+z^{2}}, \quad f^{\prime}=\frac{2 \lambda N z}{1+z^{2}}, \quad g^{\prime}=g+\frac{2 \lambda N}{1+z^{2}}$,

where

$$
z=\operatorname{ctn} \alpha,
$$

and where $\alpha$ is the angle under which the curves corresponding to the lines of curvature on $S^{\prime}$ cut the lines of curvature on $S$.

The Codazzi equations for $S^{\prime}$, by virtue of the corresponding equations (2) for $S$, can be written in the forms

$$
\frac{\partial\left(e^{\prime}-e\right)}{\partial v}=\frac{\partial f^{\prime}}{\partial u}, \quad \frac{\partial\left(q^{\prime}-g\right)}{\partial u}=\frac{\partial f^{\prime}}{\partial v} \text {. }
$$

Substituting for $e^{\prime}-e, f^{\prime}$, and $g^{\prime}-g$ their values as given by (4), and solving for $\partial z / \partial u, \partial z / \partial v$, we obtain two equations which reduce to

$$
\frac{\partial}{\partial u} \frac{z}{\lambda N}=\frac{\partial}{\partial v} \frac{1}{\lambda N}, \quad \frac{\partial}{\partial v} \frac{z}{\lambda N}=-\frac{\partial}{\partial u} \frac{1}{\lambda N} .
$$

* We have hereby assumed that $N \neq 0$. But a sphere obviously admits no surface applicable to it in the manner prescribed. 
But these equations are compatible if and only if the reciprocal of $\lambda N$ is a harmonic function.

THEOREM 1. There exists a one-parameter family of surfaces applicable to a given surface $S$ with preservation of both curvatures if and only if the lines of curvature on $S$ form an isometric system, and if, when isometric parameters for this system are introduced, the reciprocal of $\lambda\left(1 / r_{1}-1 / r_{2}\right)$ is a harmonic function.

If one function, $\psi$, to which the reciprocal of $\lambda N$ is conjugate, is obtained by a quadrature, the general solution of equations (5) is

$$
z=\lambda N(\psi+k)
$$

where $k$ is an arbitrary constant. Substituting this value of $z$ in (4), we obtain $e^{\prime}, f^{\prime}$, and $g^{\prime}$ for the surfaces $S^{\prime}$. Thus these surfaces are completely determined.

That a surface $S$ of constant mean curvature admits $\infty^{1}$ surfaces $S^{\prime}$ applicable to it with preservation of both curvatures is well known.*

It is in this case only that the angle $\alpha$ is constant and that the lines of curvature on $S$ correspond to the lines of curvature on one of the surfaces $S^{\prime}$. For, by (3), the mean curvature is constant only if $\lambda N$ is constant, and, by (5), $\lambda N$ is constant only when $z$ is constant.

3. Applicability in an Infinity of Ways. Consider now an arbitrary one-parameter family of surfaces, every pair of which are applicable with preservation of both curvatures. An obvious necessary condition that this applicability be possible in a continuous infinity of ways is that the surfaces be $W$-surfaces, i. e., that the curvatures be functionally related. It is surprising to find that, if we exclude the surfaces of constant mean curvature, this condition is also sufficient.

Theorem 2. Every pair of a one-parameter family of (isometric) $W$-surfaces satisfying the conditions of Theorem 1 , but of variable mean curvature, are applicable in a continuous infinity of ways with preservation of both curvatures.

* Cf. Bonnet, loc. cit. 
For, if we except surfaces of constant mean curvature, an isometric $W$-surface is either a surface of revolution or a surface of one of several special types. The theorem is obvious in the first case. In the second case, it follows directly from certain investigations by the author, which are to be published elsewhere.

A surface of constant mean curvature admits $\infty^{1}$ surfaces each applicable to it in a continuous infinity of ways with preservation of both curvatures if and only if it is applicable to a surface of revolution. The surfaces of constant mean curvature with this property have been determined by Raffy.*

4. Conclusion. In conclusion, we sketch the application of the method of $\S 2$ to the general problem. In this case we assume merely that the lines of curvature on $S$ are parametric. If $S^{\prime}$ is applicable to $S$ with preservation of both curvatures,

Then

$$
e^{\prime} g^{\prime}-f^{\prime 2}=e g, \quad E g^{\prime}+G e^{\prime}=E g+G e .
$$

$e^{\prime}=e-\frac{2 E N}{1+z^{2}}, \quad f^{\prime}=\frac{2 \sqrt{E G} N z}{1+z^{2}}, \quad g^{\prime}=g+\frac{2 G N}{1+z^{2}}$,

where $z=\operatorname{ctn} \alpha$, and $\alpha$ has the same significance as before.

The Codazzi equations for $S^{\prime}$, after application of those for $S$, become

$$
\begin{aligned}
& \frac{\partial\left(e^{\prime}-e\right)}{\partial v}=f^{\prime} \frac{\partial}{\partial u} \log \left(f^{\prime} \sqrt{\frac{G}{E}}\right), \\
& \frac{\partial\left(g^{\prime}-g\right)}{\partial u}=f^{\prime} \frac{\partial}{\partial v} \log \left(f^{\prime} \sqrt{\frac{E}{G}}\right) .
\end{aligned}
$$

Proceeding as in $\S 2$, we obtain finally the equations

$$
\text { (6) } \frac{\partial z}{\partial u}=A z-B \sqrt{\frac{E}{G}}, \quad \frac{\partial z}{\partial v}=B z+A \sqrt{\frac{G}{E}},
$$

* Sur certaines surfaces dont les rayons de courbure sont liés par une relation, Bulletin de LA SociÉté de France, vol. 89 (1890-91), pp. 158-169. 
where

$$
\begin{aligned}
& A=\frac{1}{N} \frac{\partial M}{\partial u}=\frac{\partial \log G N}{\partial u}, \\
& B=-\frac{1}{N} \frac{\partial M}{\partial v}=\frac{\partial \log E N}{\partial v} .
\end{aligned}
$$

But equations (6) are compatible if and only if

where

$$
P z=Q
$$

$$
\left\{\begin{array}{l}
P=\frac{\partial A}{\partial v}-\frac{\partial B}{\partial u}=\frac{\partial^{2}}{\partial u \partial v} \log \frac{G}{E} \\
Q=\frac{\partial}{\partial u}\left(A \sqrt{\frac{G}{E}}\right)+\frac{\partial}{\partial v}\left(B \sqrt{\frac{E}{G}}\right)-A^{2} \sqrt{\frac{G}{E}}-B^{2} \sqrt{\frac{E}{G}}
\end{array}\right.
$$

Theorem 3. There are $\infty^{1}$ surfaces $S^{\prime}$ applicable to a given surface $S$ with preservation of both curvatures if $P=0$, $Q=0$; there is a unique surface $S^{\prime}$ if $P \neq 0$ and $z=Q / P$ satisfies equations (6). Otherwise no surface $S^{\prime}$ exists.

According to (8), the vanishing of $P$ is the condition that $S$ be an isometric surface. If, then, isometric parameters are introduced, so that $E=G=\lambda, Q=0$ becomes the condition that $1 / \lambda N$ be a harmonic function, provided that for $A$ and $B$ the second values given in (7) are used. If the first values are substituted instead, $Q=0$ reduces to a condition more complicated in form, but involving merely the principal radii of curvature.

HARVARD UNIVERSITY 